\title{
Optimized Hybrid Model for Gaussian Noise Reduction images
}

\author{
Lubna Farhi ${ }^{1}{ }^{*}$, Agha Yasir $^{1}$, Farhan Ur Rehman ${ }^{2}$, Baqar Ali Zardari ${ }^{3}$, Ramsha Shakeel ${ }^{1}$, Samia Shakeel ${ }^{1}$ \\ ${ }^{1}$ Department of Electronic Engineering, Sir Syed University of Engineering and Technology, Karachi. \\ ${ }^{2}$ Department of Mechanical Engineering, University of Toronto, Toronto, Canada \\ ${ }^{3}$ Department of Computer Science, QUEST, Nawabshah, Pakistan. \\ ${ }^{*}$ Corresponding author: Ifarhi@ssuet.edu.pk
}

Abstract

\begin{abstract}
In this paper, image noise is removed by using a hybrid model of wiener and fuzzy filters. It is a challenging task to remove Gaussian noise (GN) from an image and to protect the image's edges. The Fuzzy-Wiener filter (FWF) hybrid model is used for optimizing the image smoothness and efficiency at a high level of GN. The efficiency is measured by using Structural Similarity (SSIM), Mean Square Error (MSE), and Peak Signal to Noise Ratio (PSNR). The proposed algorithm substitutes a mean value of the matrix for a non-overlapping block and replaces the total pixel number with each direction. In the proposed model, overall results proved that the optimized hybrid model FWF has an enormous computational speed and impulsive noise reduction, which enables efficient filtering as compared to the existing techniques.
\end{abstract}

Keywords-Gaussian noise, Weiner filter, Fuzzy filter, Noise removal, Filtering techniques

\section{Introduction}

$\mathrm{T}$ HE denoising is an essential part of processing required before it can be used for recognition or classification purposes. In this digital world, the usage of imaging in every field is becoming increasingly popular. As far as the quality of an image is concerned, noise is always present in digital images. It is basically unwanted information or unwanted data which reduces their quality and introduces blur. There are various types of noises present in digital images and anyone of these can arise during the image acquisition, transmission, coding, or processing steps [15]. The fundamental goal is to restore the original image by suppressing noise. Since filtering plays a vital role in the preprocessing of images, various types of filters have been applied to remove noise. Mathematical representation of the filtering technique is as follows [1],

$$
\begin{gathered}
G(x, y)=F(x, y) * U(x, y)+N(x, y) \\
H(x, y)=R[G(x, y)]
\end{gathered}
$$

where $F(x, y)$ is the acquired image, $U(x, y)$ is the degradation function, $*$ represents convolution,

ISSN: 2523-0379 (Online), ISSN: 1605-8607 (Print)

DOI: https://doi.org/10.52584/QRJ.1901.02

This is an open access article published by Quaid-e-Awam University of Engineering Science \& Technology, Nawabshah, Pakistan under CC BY 4.0 International License.
$N(x, y)$ shows noise, $G(x, y)$ is the initial corrupted output image and $H(x, y)$ is the final filtered output [2]. The random noise is added in the image, and it can be modeled as Gaussian or normal distribution. The probability density function (PDF) which is almost equal to the normal distribution in a statistical noise model is known as Gaussian Noise [3].

Several filtration techniques have been investigated in the literature to reduce or remove the Gaussian noise. The most common filters are Weiner filter and Fuzzy filter. The Weiner filter is one of the most popular filters in order to remove the Gaussian noise and it is usually applied in the signal processing and image processing [4]. The existing Weiner filter is effective to reduce noise, but sometimes it blurs the edges of an image because it is spatially invariant [5]. A Weiner filtering Kernel approach is mainly utilized for removing the Gaussian noise from images [6]. The disadvantage of this technique is that in the weight function it assigns the weight value to the pixels. Hence, the developed Weiner filter fails to preserve the edges of the image and as a result some of the information is lost. The switching Weiner filter is used for image denoising in [7]. Due to this drawback, this technique is only efficient for the impulse noise removal, not for continuous noise. On the other hand, 
fuzzy based filtering technique can reduce noise in a good way with expert knowledge and is suitable for specific applications in complicated scene analysis and object recognition [8]. The researcher develops fuzzy rule-based system for denoising a corrupted image. This system employs fuzzy rules for smoothing, sharpening and specially edge detection [9].

\section{Related Work}

Currently, there are several noise models in practice that are used to denoise digital images. Generally, there are two types of noise models. In additive noise model, noise signal is added with the original signal to obtain a corrupted noisy signal. In multiplicative noise model noise signal is multiplied with the original signal to produce the resultant interpreted signal [15].

Different kinds of noises have their own unique features and thus require different techniques to remove them. For example, Gaussian noise is uniformly distributed, and each pixel is the sum of true pixels in the image [14]. Salt and Pepper noise, on the other hand, is an impulse noise where it only has two distinct values: high or low. The values of the noisy pixels are either set too high or too low resulting in salt and pepper noise [16].

Since each type of noise has different features, it requires different denoising techniques to remove them. To mitigate the shortcomings of each of the individual aforementioned models, a fuzzy-Weiner filter (FWF) hybrid model is proposed for removing Gaussian noise. In the proposed model, a modified Weiner filter is used by inserting the parameters of fuzzy filter. The fuzzy filter works on all pixel directions (east, west, south, and north). However, these pixels' location ease to find every pixel at the edge. The analysis shows that the proposed algorithm improved quality of an image as compared to the existing filtering techniques.

This paper is organized as follows. Section 2 explains the proposed methodology. Experimental analysis has been done in section 3. Section 4 describes the simulated result. Section 5 concludes the paper.

\section{Methodology}

This paper investigates the enhanced filtering method by combining the existing Weiner and fuzzy filter techniques. The performance metrics SSIM, MSE and PSNR are compared with each other.

\subsection{Wiener Filter}

Wiener filter has a noise reduction kernel with spatial domains which it utilizes to eliminate the noise from images. For enhancing the image quality, the filter follows the following steps. Firstly, it selects the size of the matrix $(n \times m)$ for the noise reduction filter. Secondly, the selected matrix is used to recalculate the new pixel values compared to the degraded pixel values [10]. The Wiener filter involves both the variance and average pixel values in the $(n \times m)$ matrix and mathematically can be represented as follows,

$$
\begin{gathered}
\mu=\frac{1}{(N \times M)} \sum_{(n, m \in p)} y(n, m) \\
\sigma=\sqrt{\frac{1}{(N \times M)} \sum_{(n, m \in p)} y^{2}(n, m)}-\mu^{2}
\end{gathered}
$$

where, $\mu, \sigma,(n \times m)$ and $y(n, m)$ represents mean, variance of noise, size of the area $p$, and total number of pixels, respectively. The estimated values of the wiener filter are represented as follows.

$$
B_{w}(n, m)=\mu+\frac{\left(\sigma^{2}-v^{2}\right)}{\sigma^{2}} *[y(n, m)-\mu]
$$

Equation 5 gives a blurry image and edges are not preserved.

\subsection{Fuzzy Filter}

Let $\mathrm{F}(\mathrm{x}, \mathrm{y})$ be the input image. According to the fuzzy filtering, the output is given as follows [13] [12],

$$
B_{F}(n, m)=\frac{\sum_{(x, y)} F_{(x, y)} f_{(x, y)}}{\sum_{(x, y)} F_{(x, y)}}
$$

where, $f_{(x, y)}$ is the general 8-neighbour function, that is proportional to fuzzy membership function. The general 8- neighbor function is determined by the following function,

$$
F_{(x, y)}=\exp \left(\frac{f_{(x, y)}-f_{\max }^{2}}{2 \sigma}\right)
$$

where $f_{(\max )}$ is the maximum intensity value within 8- neighbors of a point chosen as the center value, and $\sigma$ is the standard deviation of all intensity values within the window. $F_{(x, y)}$ is calculated for the filtering window. It is more reasonable to calculate the fuzzy function reflecting the effects of 8 neighbors. Hence, to determine the degree of corruption of each pixel, we have incorporated a condition of modification to divide $F_{(x, y)}$ in Equation 7 by 8 . Since the image intensity is represented using the range of 0 to 255 , therefore, the intensity is either 0 or 255 (i.e., at the boundary) for the membership value and other intensity values. 
Therefore, the 8-neighboring function for each pixel can be determined by Equation 8 .

$$
F_{(x, y)}= \begin{cases}1, & \text { if } f_{(x, y)}=0 \text { or } 255 \\ \exp \left(-\frac{f_{(}(x, y)-f_{\max }^{2}}{2 \sigma}\right) & \text { otherwise }=0\end{cases}
$$

\subsection{Different Filter Techniques}

There has been an extensive research on denoising techniques for different types of noise. It has been presented and discussed in Table 1 below. To remove noise without excessive smoothing of important details, a denoising technique needs to be spatially adaptive. Different techniques are used depending on the noise model. In this paper, proposed FWF filter is characterized by the following assumption:

1) Signal and noise are stationary linear random processes with known spectral characteristics.

2) The filter must be physically realizable, i.e., causal.

3) Performance criteria of presented filter is minimum mean-square error and maximum PSNR.

\subsection{Proposed Fuzzy Weiner Filter (FWF)}

Our goal is to increase image quality by inserting the fuzzy parameters into the existing Weiner filter. The FWF based technique is intended to remove noise in images as shown in Figure 1. In addition, this technique preserves the edges at the highest level of Gaussian noise (HLGN). The modified algorithm, which is based on the fuzzy filter, replaces the mean value by $n \times m$ window size for non-overlapping blocking and the total number of pixels is replaced from every direction. The FMF is represented as follows:

$$
\left.B_{F M W F}(n, m)=\frac{f(x, y)}{n \times m}+\frac{\sigma^{2}-v^{2}}{\sigma^{2}} * \frac{D-f(x, y)}{n \times m}\right)
$$

Where,

$$
\begin{gathered}
D=[d N(\text { North }), d S(\text { South }) d E(\text { East }) d W(\text { West })] ; \\
d N(n, m)=y(n-1, m)-y(n, m) \\
d S(n, m)=y(n+1, m)-y(n, m) \\
d E(n, m)=y(n, m+1)-y(n, m) \\
d W(n, m)=y(n, m-1)-y(n, m)
\end{gathered}
$$

In this paper, performance metrics i.e., SSIM, MSE and PSNR are used to compare the performance of the original image $l(m, n)$ and the filtered image $k(m, n)$. SSIM is the performance metric that quantifies the

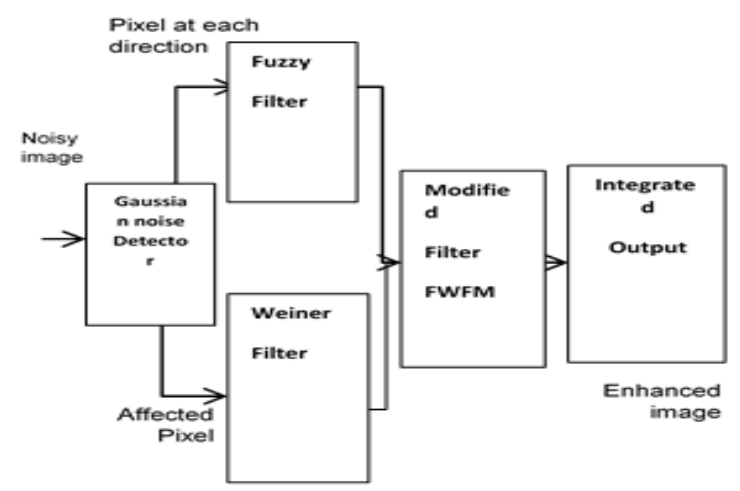

Fig. 1: Proposed system model

image quality degradation [12]. It is a method used to measure the similarity between two images. The SSIM values are between 0 and 1 , where the later is considered as a good quality image. Here, $L$ is the range which is dynamic i.e. (2bits/pixels -1 ), $K_{1}$ and $K_{2}$ are constants, i.e. 0.01 and 0.03 respectively, and $C_{1}=\left(K_{1} L\right)^{2}, C_{2}=\left(K_{2} L\right)^{2}$.

SSIM is calculated as follows.

$$
\operatorname{SSIM}_{(x, y)}=\frac{\left(2 \mu_{x} \mu_{y}+C_{1}\right)\left(2 \sigma_{x y}+C_{2}\right)}{\left(2 \mu_{x}^{2} \mu_{y}^{2}+C_{1}\right)\left(\sigma_{x}^{2}+\sigma_{y}^{2}+C_{1}\right)}
$$

MSE is the differences between the original image and the filtered image. A low value of MSE indicates lower error in the image [11] which means the quality of the image is adequate. Here $I(n, m)$ is the original image and $K(n, m)$ is the filtered image. MSE is calculated as follows.

$$
M S E=\frac{1}{M * N} \sum_{(n=0, m=0)}^{(M-1, N-1)}[I(n, m)-K(n, m)]^{2}
$$

PSNR is the ratio between the maximum power and the noise corrupting image [12]. It is also defined via the Root Mean Square Error (RMSE). As in the case of MSE, a high value of the PSNR indicates a high quality of image. The PSNR is calculated by Equation 12 ,

$$
P S N R=20 \log _{10}\left(\frac{255}{R M S E}\right)
$$

where $R M S E=\sqrt{M S E}$.

\section{Experimental Analysis}

This section of paper briefly discusses the experimental results and provides a detailed explanation of the modified Weiner filter and the performance metrics. Comparative and quantitative analysis of fuzzy modified Weiner filter (FMWF) has also been performed. 


\begin{tabular}{|l|l|l|l|}
\hline Filter & Technique & Benefits & Weaknesses \\
\hline Linear spatial filter & $\begin{array}{l}\text { Average of pixels } \\
\text { contained in neighbor } \\
\text { pixels of the filter mask }\end{array}$ & $\begin{array}{l}\text { Reduced "sharp" transitions } \\
\text { in intensities. }\end{array}$ & $\begin{array}{l}\text { Edges by sharp intensity } \\
\text { transitions will be blurred }\end{array}$ \\
\hline $\begin{array}{l}\text { Ranking the pixels in } \\
\text { neighbor pixels and } \\
\text { replacing the value of } \\
\text { center pixel by ranking } \\
\text { result }\end{array}$ & $\begin{array}{l}\text { Quite effective against } \\
\text { the impulse noise } \\
\text { Beighted averaging filter, } \\
\text { distance and the intensity } \\
\text { distance with respect to the } \\
\text { center pixel }\end{array}$ & $\begin{array}{l}\text { Ability to preserve edges } \\
\text { while doing spatial smoothing. }\end{array}$ & $\begin{array}{l}\text { Half values are greater } \\
\text { staircase effect and single resolution } \\
\text { in nature }\end{array}$ \\
\hline Wavelet transform & $\begin{array}{l}\text { Set of orthonormal basis } \\
\text { functions generated by dilation } \\
\text { and translation of scaling }\end{array}$ & $\begin{array}{l}\text { Preserving the signal } \\
\text { characteristics, regardless } \\
\text { of its frequency content }\end{array}$ & $\begin{array}{l}\text { Thresholding functions, selection } \\
\text { is hard to achieve better results }\end{array}$ \\
\hline
\end{tabular}

TABLE 1: Comparison of the existing techniques

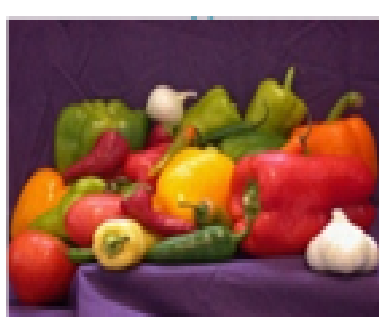

(a)

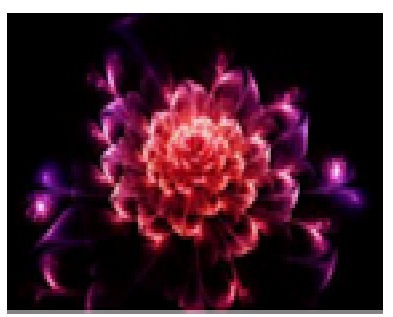

(b)

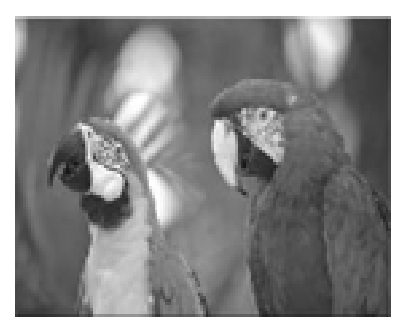

(c)

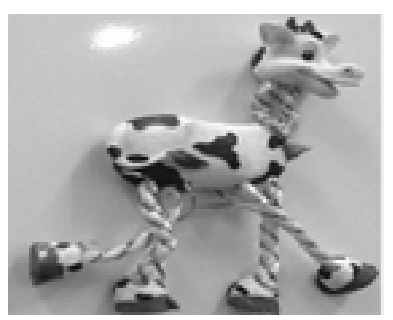

(d)

Fig. 2: Original images

\subsection{Experimental Setup}

The FWF hybrid model was implemented in MATLAB with 4GB RAM, Intel core i5. The performance of the proposed technique is compared with existing techniques to ascertain its efficacy in terms of the performance metrics.

\subsection{Quantitative Analysis}

In this section, four images, as shown in Figure 2, are utilized to analyze the performance of the filters. In this study, performance evaluation is validated by calculating the SSIM, MSE and PSNR parameters as shown in Table 2, 3 and 4 . The performance evaluation is defined for random images with different variance $(\sigma)$ of Gaussian Noise. In Figure 3(b), 4(b) and 5(b), the performance of the proposed model is measured by adding $\sigma=0.01,0.04$ and 0.1 of Gaussian noise in the original image.

\subsection{Comparative Analysis}

Existing filters need a lot of edge data for image denoising which is scarcely available in images. In this study, a FWF model is proposed for distinguishing the edge information of images. In Table 5, 6 and

\begin{tabular}{|l|c|l|l|}
\hline \multirow{2}{*}{ Image } & \multicolumn{3}{|c|}{ Gaussian Noise $\sigma=0.01$} \\
\cline { 2 - 4 } & SSIM & MSE & PSNR \\
\hline $\mathrm{A}$ & 0.77061 & 28.707764 & 87.559638 \\
\hline $\mathrm{B}$ & 0.80170 & 30.767076 & 54.497024 \\
\hline $\mathrm{C}$ & 0.26806 & 43.60974 & 20.1296 \\
\hline $\mathrm{D}$ & 0.23778 & 45.4913 & 19.1186 \\
\hline
\end{tabular}

TABLE 2: FWF filter performance at $\sigma=0.01$

\begin{tabular}{|l|l|l|l|}
\hline \multirow{2}{*}{ Image } & \multicolumn{3}{|l|}{ Gaussian Noise $\sigma=0.04$} \\
\cline { 2 - 4 } & SSIM & MSE & PSNR \\
\hline $\mathrm{A}$ & 0.789043 & 29.755500 & 80.509008 \\
\hline $\mathrm{B}$ & 0.918900 & 30.960096 & 53.998564 \\
\hline $\mathrm{C}$ & 0.333831 & 23.2033 & 14.5094 \\
\hline $\mathrm{D}$ & 0.298718 & 34.0393 & 15.3061 \\
\hline
\end{tabular}

TABLE 3: FWF filter performance at $\sigma=0.04$

\begin{tabular}{|l|l|l|l|}
\hline \multirow{2}{*}{ Image } & \multicolumn{3}{|l|}{ Gaussian Noise $\sigma=0.01$} \\
\cline { 2 - 4 } & SSIM & MSE & PSNR \\
\hline $\mathrm{A}$ & 0.927880 & 31.229870 & 79.2355509 \\
\hline $\mathrm{B}$ & 0.969900 & 35.990001 & 51.7785903 \\
\hline $\mathrm{C}$ & 0.844300 & 47.838000 & 11.3671 \\
\hline $\mathrm{D}$ & 0.572980 & 45.658000 & 11.5697 \\
\hline
\end{tabular}

TABLE 4: FWF filter performance at $\sigma=0.1$ 


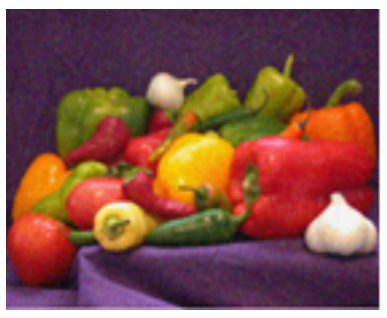

(a)

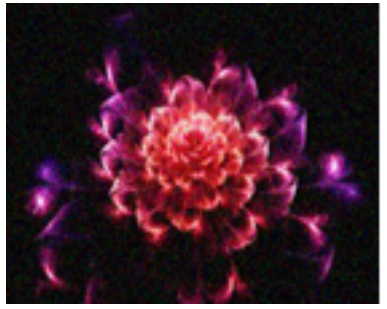

(e)

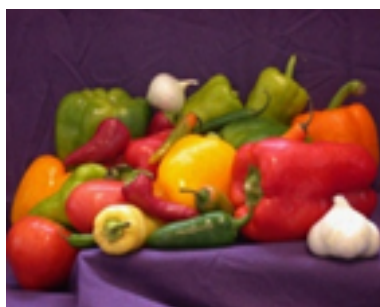

(b)

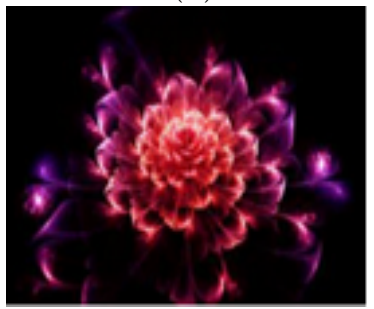

(f)

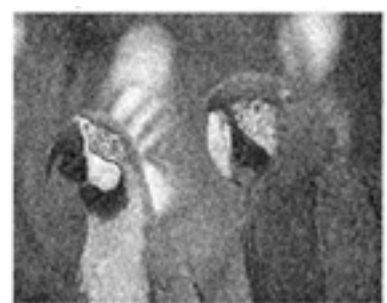

(c)

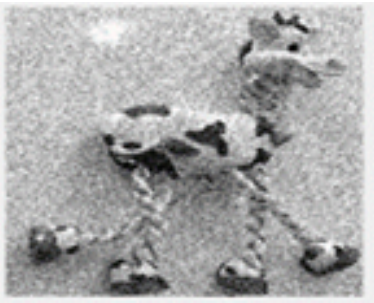

$(\mathrm{g})$

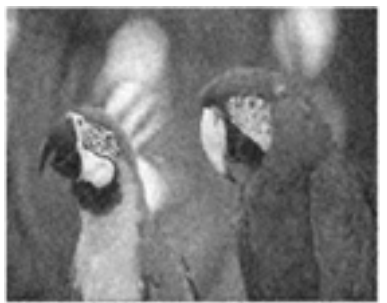

$(\mathrm{d})$

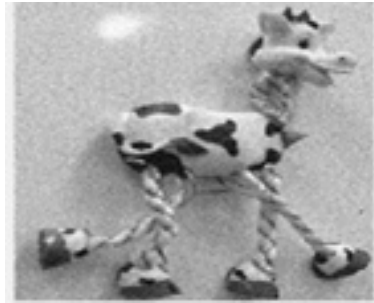

(h)

Fig. 3: Images with $\sigma=0.01$ (a, c, e, g), denoised images (b, d, f, h)

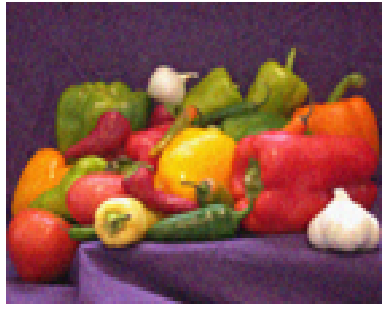

(a)

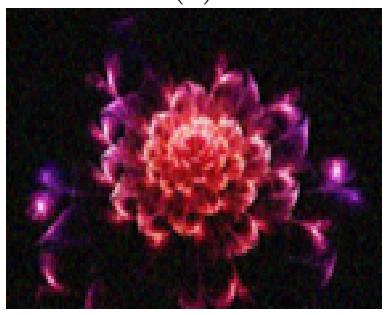

(e)

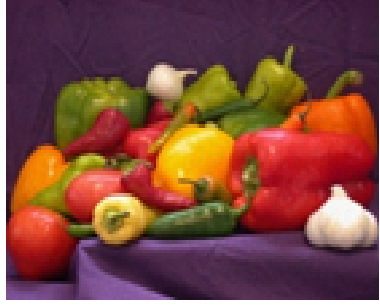

(b)

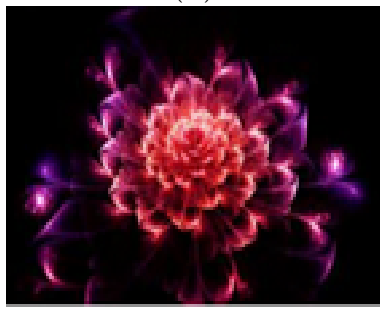

(f)

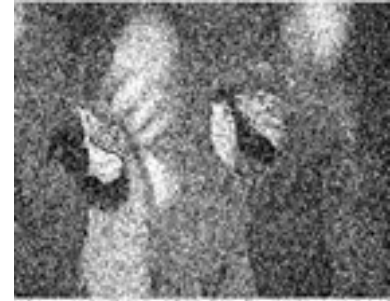

(c)

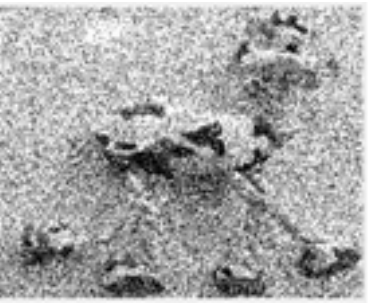

(g)

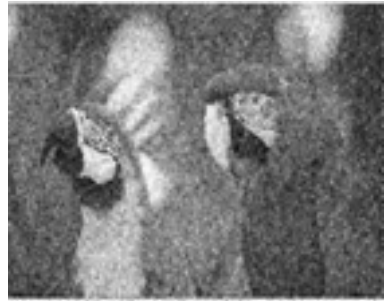

(d)

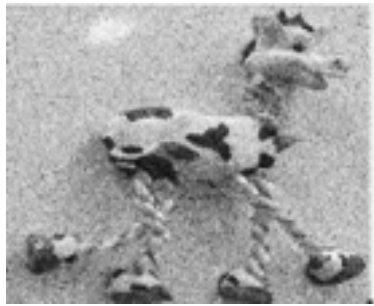

(h)

Fig. 4: Images with $\sigma=0.04$ (a, c, e, g), denoised images (b, d, f, h)

7, the comparative analysis is performed between the presented hybrid model and the traditional filters, i.e., Weiner and Fuzzy filters. The analysis is done by the performance evaluation parameters such as SSIM, MSE and PSNR as shown in Fig. 6, 7 and 8. The efficiency of the presented filter is verified by adding the Gaussian Noise $\sigma=0.1,0.04$ and 0.1 . The existing filters work classically in the noisy pixels as compared to proposed filter.

\section{Result \& Discussion}

The quality of an image is degraded when noise is introduced in the image during transmission and acquisition processes. Therefore, improving the image quality is very essential in numerous applications like object detection, image retrieval, biometric verification, etc. In this research, the proposed model (FMF) is used to reduce noise and retain the edge information in digital images. The analysis is done by the performance evaluation parameters such as SSIM, MSE and PSNR. The proficiency of the proposed filter and the existing filters are validated under different levels of Gaussian noise. From the simulated results, the proposed model showed 0.5 to 0.8 improvement in SSIM, while the error rate (MSE) reduced about 10 to 12, and there was 15 to $20 \mathrm{~dB}$ enhancement in PSNR. The main advantage and the key benefit of this proposed (FMF) filter is that it is more robust against Gaussian noise 


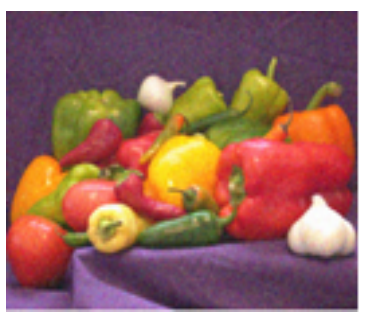

(a)

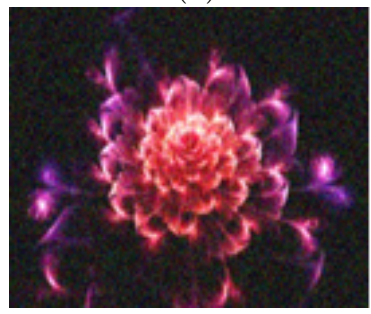

(e)

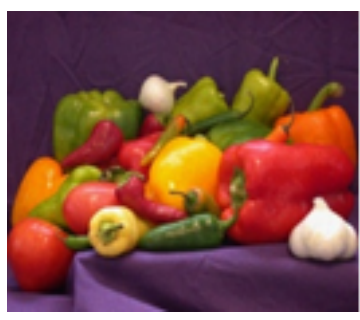

(b)

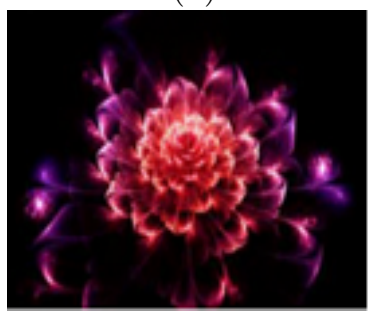

(f)

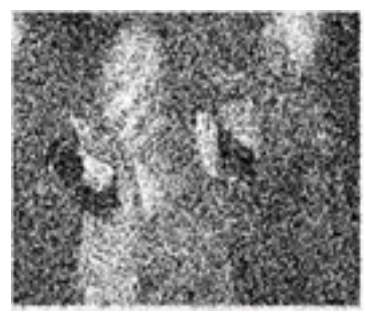

(c)

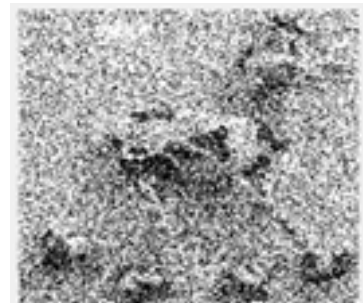

(g)

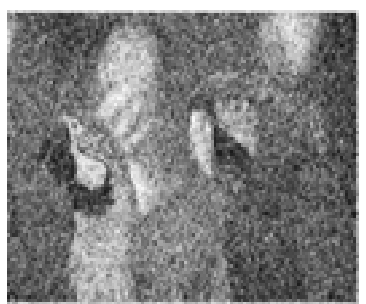

(d)

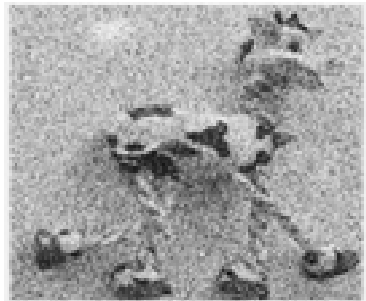

(h)

Fig. 5: Images with $\sigma=0.01$ (a, c, e, g), denoised images (b, d, f, h)

\begin{tabular}{|l|l|l|l|l|}
\hline \multirow{2}{*}{ Figure } & \multirow{2}{*}{ Filter } & GN $\sigma=0.01$ & GN $\sigma=0.04$ & GN $\sigma=0.1$ \\
\cline { 3 - 5 } & & \multicolumn{3}{|c|}{ SSIM } \\
\hline \multirow{3}{*}{$\mathrm{A}$} & Wiener & 1.27 & 1.39 & 1.45 \\
\cline { 2 - 5 } & Fuzzy & 1.00 & 1.10 & 1.08 \\
\cline { 2 - 5 } & Proposed & $\mathbf{0 . 7 7}$ & $\mathbf{0 . 7 8}$ & $\mathbf{0 . 9 2}$ \\
\hline \multirow{3}{*}{$\mathrm{B}$} & Wiener & 1.24 & 1.12 & 1.46 \\
\cline { 2 - 5 } & Fuzzy & 1.03 & 1.01 & 1.19 \\
\cline { 2 - 5 } & Proposed & $\mathbf{0 . 9 4}$ & $\mathbf{0 . 8 6}$ & $\mathbf{0 . 9 9}$ \\
\hline
\end{tabular}

TABLE 5: Comparative analysis of SSIM

\begin{tabular}{|l|l|l|l|l|}
\hline \multirow{2}{*}{ Figure } & \multirow{2}{*}{ Filter } & GN $\sigma=0.01$ & GN $\sigma=0.04$ & GN $\sigma=0.1$ \\
\cline { 3 - 5 } & & \multicolumn{3}{|c|}{ MSE } \\
\hline \multirow{3}{*}{ A } & Weiner & 43.22 & 46.89 & 48.98 \\
\cline { 2 - 5 } & Fuzzy & 33.05 & 36.11 & 39.56 \\
\cline { 2 - 5 } & Proposed & $\mathbf{2 8 . 7 0}$ & $\mathbf{2 9 . 7 5}$ & $\mathbf{3 1 . 2 2}$ \\
\hline \multirow{3}{*}{$\mathrm{B}$} & Weiner & 43.20 & 41.57 & 44.01 \\
\cline { 2 - 5 } & Fuzzy & 37.99 & 38.09 & 40.02 \\
\cline { 2 - 5 } & Proposed & $\mathbf{3 0 . 7 6}$ & $\mathbf{3 0 . 9 6}$ & $\mathbf{3 5 . 9 9}$ \\
\hline
\end{tabular}

TABLE 6: Graphical comparison of MSE

\begin{tabular}{|l|l|l|l|l|}
\hline \multirow{2}{*}{ Figure } & \multirow{2}{*}{ Filter } & GN $\sigma=0.01$ & GN $\sigma=0.04$ & GN $\sigma=0.1$ \\
\cline { 3 - 5 } & & \multicolumn{3}{|c|}{ PSNR (dB) } \\
\hline \multirow{3}{*}{ A } & Weiner & 60.34 & 54.12 & 48.98 \\
\cline { 2 - 5 } & Fuzzy & 79.99 & 68.99 & 67.12 \\
\cline { 2 - 5 } & Proposed & 87.55 & 80.50 & 79.23 \\
\hline \multirow{3}{*}{ B } & Weiner & 38.98 & 37.56 & 32.90 \\
\cline { 2 - 5 } & Fuzzy & 46.78 & 43.12 & 42.09 \\
\cline { 2 - 5 } & Proposed & 54.49 & 53.99 & 51.77 \\
\hline
\end{tabular}

TABLE 7: Comparative analysis of PSNR 


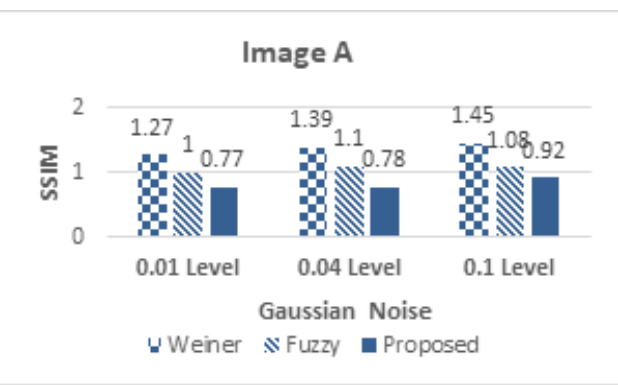

(a)

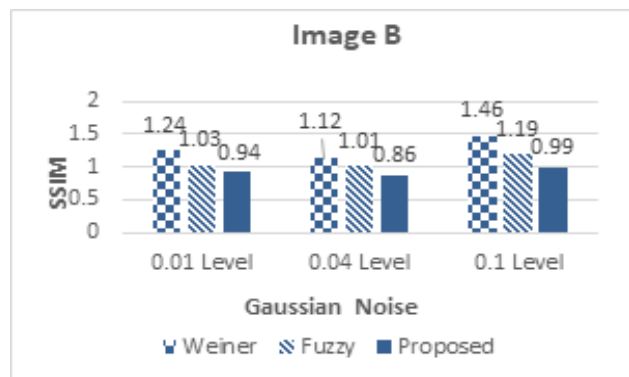

(b)

Fig. 6: Graphical comparison of SSIM

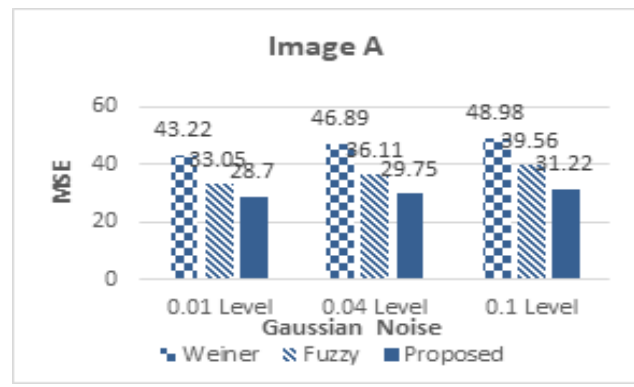

(a)

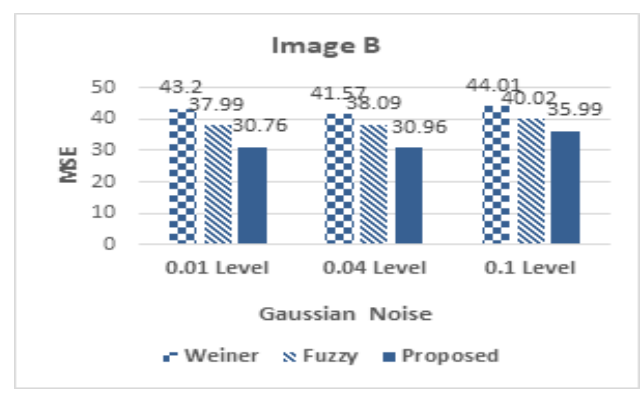

(b)

Fig. 7: Graphical comparison of MSE

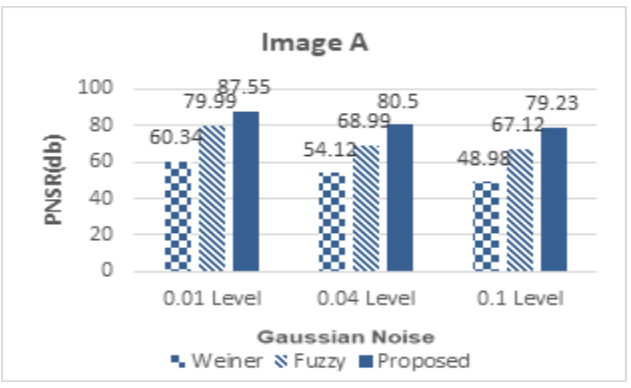

(a)

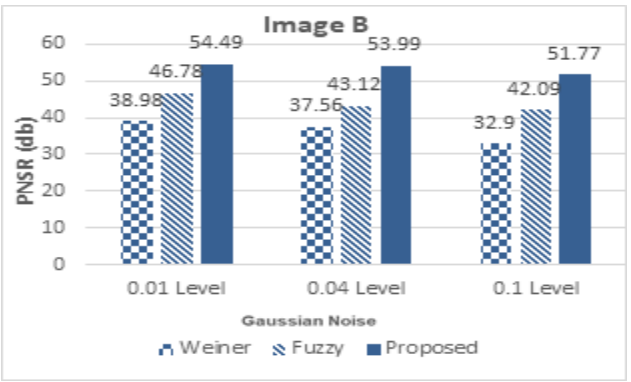

(b)

Fig. 8: Graphical comparison of PSNR

and efficiently preserves the edges of the digital image. From the experimental simulation, the proposed filter (FMF) shows the best recovery and restoration of the noisy image. The presented hybrid filter model has high accuracy even for visually challenging images. For example, as shown in Figure 3,4 and 5(b), in the fruits image, despite the different values of Gaussian noise, the edges are well-defined, and the background is smoother. Moreover, in the flowers image, the texture of the curtain and table-cloth is closer to the original image after noise removal. Lastly, in the Dog and the Parrot images, the feathers around the head of the parrots have more details; whereas, the mouth opening in the dog image is clearer than others.

The proposed algorithm more effectively overcomes the staircase effect, preserves the thin lines better, refines the weak edges and fine details, and is also more efficient in removing noise and aliasing around edges. This is shown in the GUI bivariate histogram Figure 9, 10 of the noisy and filtered images, respectively. The output histogram shows that all the intensity values are distributed evenly, enhancement is achieved, and the image is also brightened at the output as shown in Figure 10.

To conclude, the visual and quantitative results have demonstrated that the quality of the restored images by FWF method is superior and more robust as compared to other techniques.

Figure 11 shows the analysis of the results to 


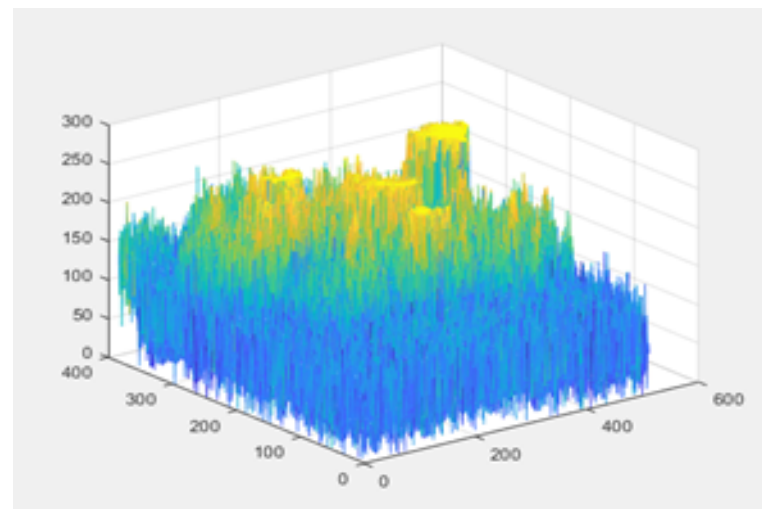

Fig. 9: GUI representation of the noisy image

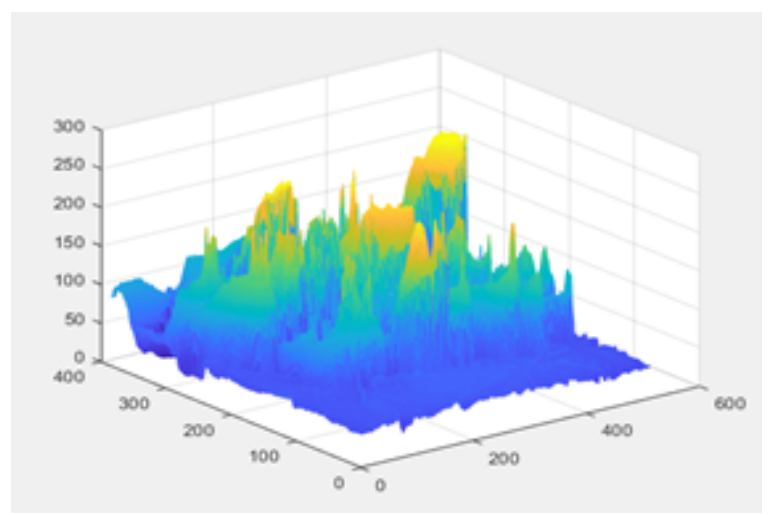

Fig. 10: GGUI representation of the proposed filtered image

authenticate the level of efficiency of the proposed filter during operation.

\section{Conclusion}

Images that contain a high amount of noise suffer especially in digital image processing. To address this issue, various algorithms, techniques, and schemes

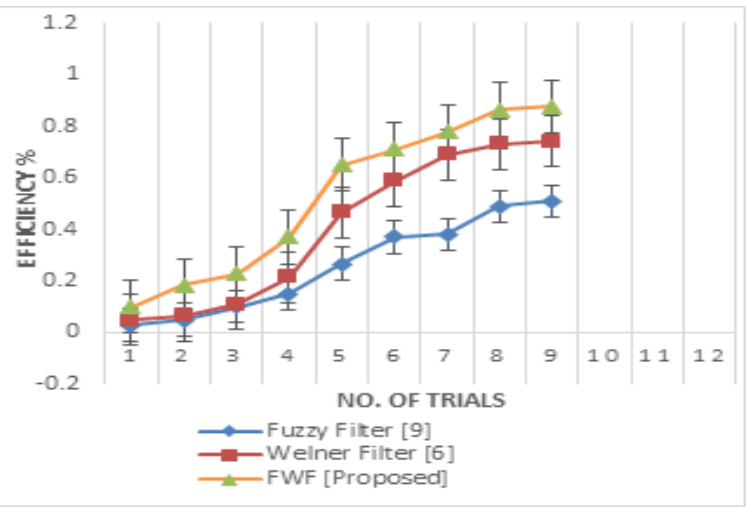

Fig. 11: Comparison between existing filters and the proposed filters have been established for many applications like image analysis, pattern recognition, etc. In this paper, a hybrid model (FWF) is proposed for removing noise in images. For checking the dynamic characteristics of the proposed model, images are simulated with various Gaussian noise variance's levels. Results comparison was then performed between the existing filters. The results show that the proposed model has significantly improved performance. This was determined by observing the SSIM, MSE and PSNR. The FWF hybrid model has achieved 0.5 to 0.8 SSIM, reduced MSE about 10 to $12 \%$, and improved PSNR by 15 to 20 $\mathrm{db}$. The main benefit of the proposed filter is that it is more vigorous against Gaussian noise and efficiently preserves the edges of the image and gives best retrieval of noisy image for compression, transmission, and recognition.

\section{References}

[1] A. Sharma and J. Singh, "Image denoising using spatial domain filters: A quantitative study, "International Congress on Image and Signal Processing (CISP), Hangzhou, 2018, pp. 293-298, 2013.

[2] Chan Rok Park, Seong- Hyeon Kang, Youngjin Lee, "Median modified wiener filter for improving the image quality of gamma camera images," Nuclear Engineering and Technology, vol 52, pp. 2328-2333, 2020.

[3] T. Bhattacharya and A. Chatterjee, "Evaluating performance of some common filtering techniques for removal of Gaussian noise in images," IEEE International Conference on Power, Control, Signals, and Instrumentation Engineering (ICPCSI), Chennai, pp. 1981-1984, 2017.

[4] S. Khetkeeree and S. Liangrocapart, "Image Restoration Using Optimized Weiner Filtering Based on Modified Tikhonov Regularization," IEEE 4th In-ternational Conference on Signal and Image Processing (ICSIP), Wuxi, China, pp. 1015-1020, 2019.

[5] V. V. Sazonov, M. A. Shcherbakov and V. A. Vasilyev, "Modified Wiener filter," International Conference on Biomedical Engineering and Computational Technologies (SIBIRCON), Novosibirsk, pp. 193-196, 2015.

[6] Y. Zhang, J. Xiao, J. Peng, Y. Ding, J. Liu, Z. Guo, and X. Zong, "Kernel Wie-ner Filtering Model with Low-Rank Approximation for Image Denoising," Information Sciences, vol. 462, pp.402-416, 2018.

[7] X. Zhang, "Image denoising using local Wiener filter and its method noise," Optic-International Journal for Light and Electron Optics, vol.127, pp.6821-6828, 2016.

[8] M. Chowdhury, Junbin Gao and R. Islam, "Fuzzy logicbased filtering for image de-noising," IEEE International Conference on Fuzzy Systems, Van-couver, pp. 2372-2376, 2016.

[9] M.H. Chowdhury, M. E. Islam, N. Begum, and M.A. Bhuiyan, "Digital Image Enhancement with Fuzzy Rulebased Filtering," 10th International Conference on Computer and Information Technology, Dhaka, pp. 250-252, 2008.

[10] S. Esakkirajan, T. Veerakumar, Subramanyam, C.H. Prem Chand, "Re-moval of high-density Salt and pepper noise through modified decision based Unsymmetrical trimmed 
median filter," IEEE Signal Process, Lett. 18, pp. 287-290, 2011.

[11] Asamoah, Dominic \& Oppong, Emmanuel \& Oppong, Stephen \& Dan-so, Juliana, "Measuring the Performance of Image Contrast Enhancement Technique," International Journal of Computer Applications, vol.181, pp. 6-13, 2018.

[12] Al Zahir, S., \& Kashanchi, F. "A new image quality measure," 26th IEEE Canadian Conference on Electrical and Computer Engineering (CCECE), 2013.

[13] R. Marudhachalam and Gnanambal Ilango, "Fuzzy Hybrid Filtering Tech-niques for Removal of Random Noise from Medical Images," International Journal of Computer Applications, vol. 38, no. 1, January 2012.

[14] Islam, M. T., Rahman, S. M., Ahmad, M. O., \& Swamy, M. N. S.," Mixed Gaussian-impulse noise reduction from images using convolutional neural network. Signal Processing: Image Communication, vol. 68, pp. 26-41, 2018.

[15] Guan, Juntao, Rui Lai, Ai Xiong, Zesheng Liu, and Lin GU. "Fixed pattern noise reduction for infrared images based on cascade residual attention CNN." Neurocomputing 377, pp. 301-313, 2020.

[16] Duarte-Salazar, Carlos A., Andrés Eduardo CastroOspina, Miguel A. Becerra, and Edilson Delgado-Trejos. "Speckle noise reduction in ultrasound images for improving the metrological evaluation of biomedical applica-tions: an overview." IEEE Access 8, pp. 15983-15999, 2020. 